\title{
Nonstationary Stochastic Resonance in a Single Neuron-Like System
}

\author{
Redouane Fakir \\ Peter Wall Institute for Advanced Studies \\ Cosmology Group, Department of Physics 83 Astronomy \\ Department of Interdisciplinary Studies \\ University of British Columbia, 6224 Agriculture Road, Vancouver, B. C. V6T 1Z1, CANADA
}

\begin{abstract}
Stochastic resonance holds much promise for the detection of weak signals in the presence of relatively loud noise. Following the discovery of nondynamical and of aperiodic stochastic resonance, it was recently shown that the phenomenon can manifest itself even in the presence of nonstationary signals. This was found in a composite system of differentiated trigger mechanisms mounted in parallel, which suggests that it could be realized in some elementary neural networks or nonlinear electronic circuits. Here, we find that even an individual trigger system may be able to detect weak nonstationary signals using stochastic resonance. The very simple modification to the trigger mechanism that makes this possible is reminiscent of some aspects of actual neuron physics. Stochastic resonance may thus become relevant to more types of biological or electronic systems injected with an ever broader class of realistic signals.
\end{abstract}


One of the remarkable aspects of stochastic resonance ${ }^{1-10}$ is the possibility of enabling the detection of a weak signal by adding noise to the input. An important recent development in this field - the discovery of nondynamical stochastic resonance ${ }^{11-13}$ - is that the phenomenon can occur independently of any details of nonlinear dynamics in the system, although the nonlinearity itself is essential for the phenomenon to occur. Thus, stochastic resonance was shown to arise in extremely simple trigger systems. Equally important was the discovery of aperiodic stochastic resonance ${ }^{14-23}$, that is, the realization that the signals made detectable by the addition of noise need not be periodic. This opened the door for investigating the occurrence of the phenomenon under a broader set of realistic conditions. More recently still, a study originally motivated by new prospects in gravitational wave detection ${ }^{24-26}$ showed that stochastic resonance can manifest itself not only when the signal is aperiodic, but also when it is markedly nonstationary ${ }^{27}$. This could potentially extend the relevance of stochastic resonance to a larger class of biological and electronic applications. The system that was shown to exhibit nonstationary stochastic resonance was a simple, nondynamical, multi-level trigger, more precisely, a summing network of differentiated single-threshold systems. Here, we report that nonstationary stochastic resonance can also manifest itself in a system as elementary as an individual, single-level trigger, provided a very simple modification is applied to the trigger mechanism. This modified single-threshold system is reminiscent of certain aspects of neuronal biophysics, and we shall briefly allude to that possible connection further below.

Consider then the single-threshold trigger mechanism that is at work in Figs.1,2. Starting with Fig.1a, it shows the input consisting of a subthreshold deterministic signal (see Fig.1b) buried in loud (i.e. above-threshold) random noise. The latter is taken to be a low-pass filtered, zero-mean Gaussian white noise. The total input frequently exceeds the threshold, resulting in the firings of Figs.2, although the deterministic signal itself never exceeds the threshold, and hence would not be detectable if it were the only input.

The injected deterministic signal is markedly nonstationary, while always remaining sub-threshold. Hence, no response would result in Figs.2 in the absence of noise. In the presence of noise, not only one does obtain a response, but the hidden deterministic signal can be easily detected in that response, as can be seen most clearly from Fig.2c. It is the strong correlation of the noise-induced response with the sub-threshold signal (Figs.3,4) that permits 
one to say that the addition of noise has allowed the detection of an otherwise undetectable signal. Note that, if the signal has more high-frequency structure than shown here, that could be dealt with by first applying the techniques of aperiodic stochastic resonance ${ }^{14-23}$ to the "straightened out" (low-frequency filtered) version of the signal. In the present letter, we focus on the nonstationary aspect of the signal, which is essentially a low-frequency characteristic.

The trigger system, in its simplest form, is sensitive only to whether (not to by how much) the threshold is exceeded. Hence, one does not expect that a single-trigger system would help efficiently with the detection of a strongly nonstationary signal. This is indeed confirmed in Fig.3a, where the response of the unmodified trigger system is shown to have little visible structure and to correlate poorly with the hidden deterministic signal.

This eventual inability of the simplest single-trigger system to help efficiently with the detection of a strongly nonstationary signal can sometimes be remedied if several such triggers are available and if their outputs can be summed ${ }^{27}$. That not withstanding, it is shown in Figs.2b,c that even one individual trigger can help achieve detection, provided that either one of the two following straightforward modifications can be made:

1) The system fires only every time interval equal to $B$, and the height of the pulse fired is proportional to the number of times the threshold has been crossed during $B$. Equivalently, the system outputs after every time interval $B$ the average of the "blind" response of Fig.2a. This situation can easily arise when, e.g., the response of a system is (by choice or by constraint) slower than the input sampling rate. Indeed, when the system is one under biological or electronic control, efficiency dictates that the output firing rate should be slower than the input sampling rate. Fig.2b shows that this modification can improve even visually the correlation with the hidden deterministic signal, which improvement is confirmed more quantitatively in Fig.3.

2) The internal dynamics of the system effects the response in a way that can be modeled by a convolution window running through the pulsed "blind" response (i.e. through Fig.2a.) This is indeed expected to be the case if the system simulated is, e.g., a cortical or a sensory neuron ${ }^{16,28-32}$. The minimal lapse between two neural firings can be as small as a few milliseconds, but there is an additional, longer time scale effecting the response, a time scale that can vary from about 10 milliseconds for certain cortical neurons, to 
about 100 milliseconds for sensory neurons. This effective integration time reflects the characteristics of the flow of ionic transmitter substances through dendritic synapses and the associated growth of the polarizing potentials involved in the neural firings. In Fig.2c, the system has been modified to reflect in the simplest way possible the basic aspects of neuron physics just

mentioned: An effective running window, taken here to be a Gaussian of width $B$, is applied to the raw pulse train of Fig.2a (i.e., the actual response is the convolution of the blind response of Fig.2a with a Gaussian of width $B$.) For most values of $B$, the response of this modified trigger is extremely well correlated to the hidden deterministic signal (see Fig.3). Simulations show that the results remain virtually unchanged for most reasonable choices of a running window. This implies that if a given type of neuron is actually found to display the phenomenon suggested by these simulations, the same could be suspected to hold for most other types of neurons.

To summarize, it can be seen from Fig.2b and Fig.2c that elementary, experimentally motivated modifications of the single-trigger system can produce a dramatic increase in the efficiency of signal detection through stochastic resonance. The implication is that even one individual neuron, or an analogous nonlinear electronic device, could help achieve the detection of nonstationary, sub-threshold signals in a noisy environment.

Coming in the wake of the remarkable leaps in the field brought about by nondynamic and aperiodic stochastic resonance, and following the recently suggested generalization to markedly nonstationary cases, the possibility seems to become ever more real that some of the simplest systems conceivable may be able to detect weak signals of an almost arbitrary nature.

\section{Acknowledgements}

I am grateful to L.M. Ward for helping me become better acquainted with neural processes. I am also grateful to W.G. Unruh for being the first to bring the phenomenon of stochastic resonance to my attention, and to B. Bergersen for helping me familiarize myself with previous literature on stochastic resonance. I have also benefited from extensive logistical support by the General Relativity \& Cosmology Group in the Department of Physics, 
University of British Columbia, by the Department of Interdisciplinary Studies at UBC and by T.E. Vassar during the preparation of this paper. This work was supported by the PWIAS of UBC and NSERC of Canada. 


\section{REFERENCES}

1. Benzi R., Sutera S. \& Vulpiani A, J. Phys. A14, L453 (1981).

2. Nicolis C., Tellus 34, 1 (1982).

3. Benzi R., Parisi G., Sutera A \& Vulpiani A., Tellus 34, 10 (1982).

4. Wiesenfeld K. \& Moss F., Nature 373, 33 (1995).

5. Nicolis C., J. Stat. Phys. 70, 3 (1993).

6. Longtin A., Bulsara A. \& Moss F, Phys. Rev. Lett.67, 656 (1991).

7. Douglass J.K., Wilkens L., Pantazelou E. \& Moss F., Nature 365, 337 (1993).

8. Bezroukov S.M. \& Vodyanoy I., Nature 378, 362 (1995).

9. Fauve S. \& Heslot F., Phys. Lett. A97, 5 (1983).

10. Mantegna R.N. \& Spagnolo B., Phys. Rev. E49 R1792 (1994).

11. Jung P., Phys. Rev. E50, 2513 (1994); Phys. Lett. A207, 93 (1995).

12. Wiesenfeld K., Pierson D., Pantazelou E., Dames C. \& Moss F., Phys. Rev. Lett. 72, 2125 (1994).

13. Gingl Z., Kiss L.B. \& Moss F., Europhys. Lett. 29, 191 (1995).

14. Collins J.J., Chow C.C. \& Imhoff T.T., Phys. Rev. E52, R3321 (1995).

15. Collins J.J., Chow C.C., Capela A.C. \& Imhoff T.T., Phys. Rev. E54, 5575 (1996).

16. Collins J.J., Imhoff T.T. \& Grigg P., J. of Neurophysiology 76, 642 
(1996).

17. Collins J.J., Chow C.C. \& Imhoff T.T., Nature 376, 236 (1995).

18. Levin J.E. \& Miller J.P., Nature 380, 165 (1996).

19. Heneghan C., Chow C.C., Collins J.J., Imhoff T.T., Lowen S.B. \& Teich M.C., Phys. Rev. E54, R2228 (1996).

20. Gailey P.C., Neiman A., Collins J.J. \& Moss F., Phys. Rev. Lett. 79, 4701 (1997).

21. Neiman A., Schimansky-Geier L. \& Moss F., Phys. Rev. E56, 9 (1997).

22. Pantazelou E., Moss F. \& Chialvo D., in Noise in Physical Systems and 1/f Fluctuations, (eds Handel P.H. and Chung A.L.) 549-552 (American Institute of Physics Press, New York, 1993.)

23. Moss F. \& Pei X., Nature 376, 221 (1995).

24. Fakir R., Int. J. Mod. Phys. D6, 49 (1997); Phys. Rev. D 50, 3795 (1994); Astrophys. J. 426, 74 (1994).

25. Unruh W.G., private communication (1995).

26. Fakir R., "A macroscopic gravity wave effect;" Los Alamos Physics Archives astro-ph/9601127.

27. Fakir R., "Nonstationary Stochastic Resonance;" to appear in Phys. Rev. E (1998).

28. Luce R.D. \& Green D.M., Psychological Review 79, 14 (1972).

29. Ward L.M., Perception 6 Psychophysics 50, 117-128 (1991).

30. Hecht S., Schlaer S. \& Pirenne M.H., J. of General Physiology 25, 819840 (1942). 
31. Seamans J.K., Gorelova, N. \& Yang, C.R. J. Neuroscience 17, 59365948 (1997).

32. König P., Engel A.K. \& Singer W. Trends in Neuroscience 19, 130-137 (1996). 


\section{Figure captions}

\section{Figure 1}

a) The input consists of a sub-threshold deterministic signal (Fig.1b) buried in random noise. Here as throughout the simulations, the total integration time is normalized to one.

b) The deterministic signal is markedly nonstationary, while always remaining sub-threshold. No response would result in Figs.2 without the presence of noise. 


\section{Figure 2}

a) Response of the unmodified trigger system, showing little visible structure and correlating poorly to the hidden deterministic signal.

b) This is the response from an individual trigger system that averages its raw pulse train (the firings of Fig.2a) every time interval B. Equivalently, this would be the response from a system that, after every time interval $B$, fires a pulse with a height proportional to the number of times during $B$ that the threshold has been exceeded.

c) The single-trigger system has been modified here in keeping with some basic facts about internal neuron physics (see text). A Gaussian running window of width $B$ is applied to the raw pulse train of Fig.2a. For most values of $B$, the response is strongly correlated to the hidden deterministic signal (see Fig.1b). 


\section{Figure 3}

This shows the correlation coefficient, which is the value of the normalized correlation function (between the response and the signal) at zero-lag, as a function of $B$, the size of the averaging bin or of the running window. The very high correlations achieved for certain values of $B$ confirm the visual detection of the deterministic signal (Fig.1b) in the responses of Figs.2b,c. 
Figure 4

The correlation coefficient (see caption of Fig.3) as a function of $\sigma$, the rms of the noise, which is divided here by threshold height of Figs.1a,b. The system clearly displays stochastic resonance. 
Figure 1a

Raw data: nonstationary sub-threshold signal hidden in random noise

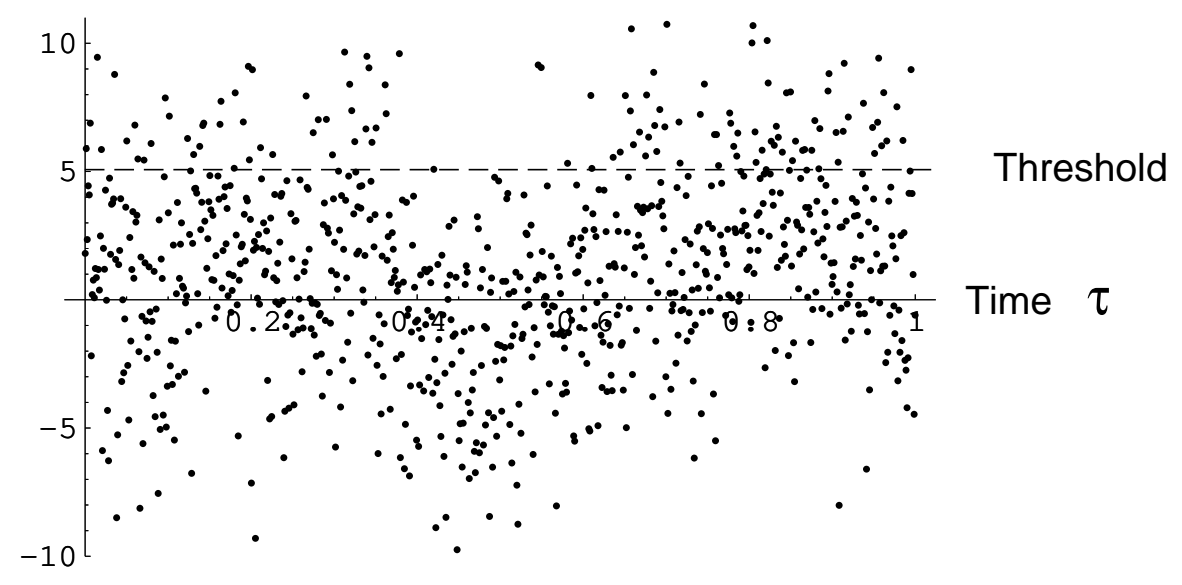


Figure 1b

Nonstationary sub-threshold signal

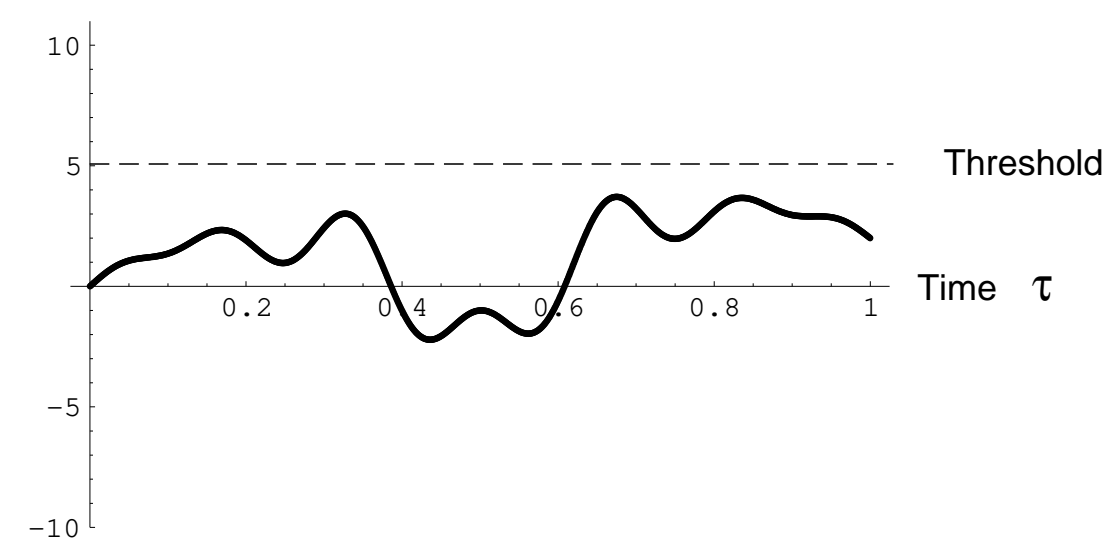


Figure 2a

Response of unmodified trigger system

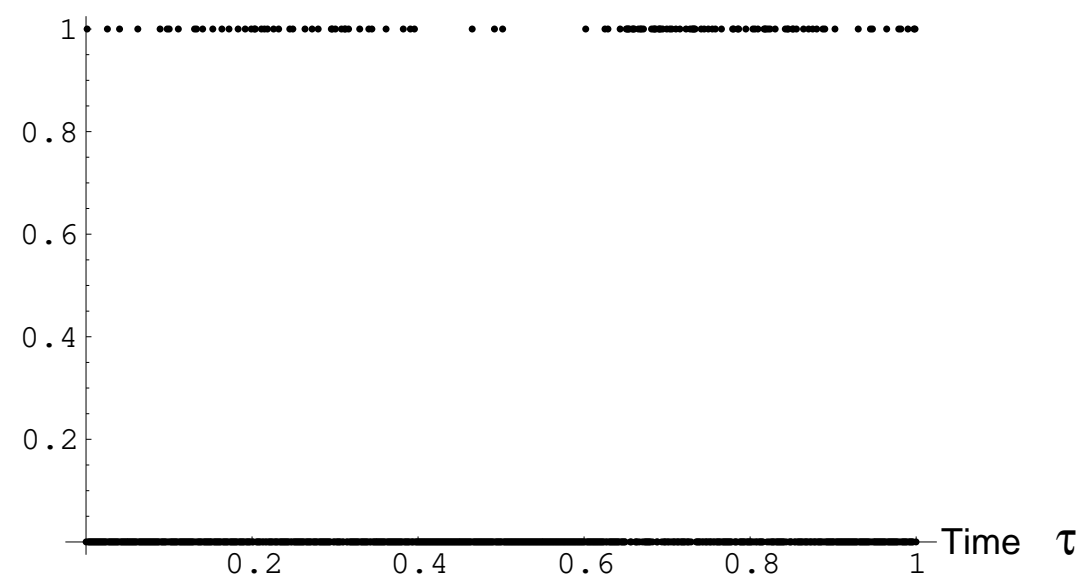


Figure 2b

Response of modified trigger system: bin averaging

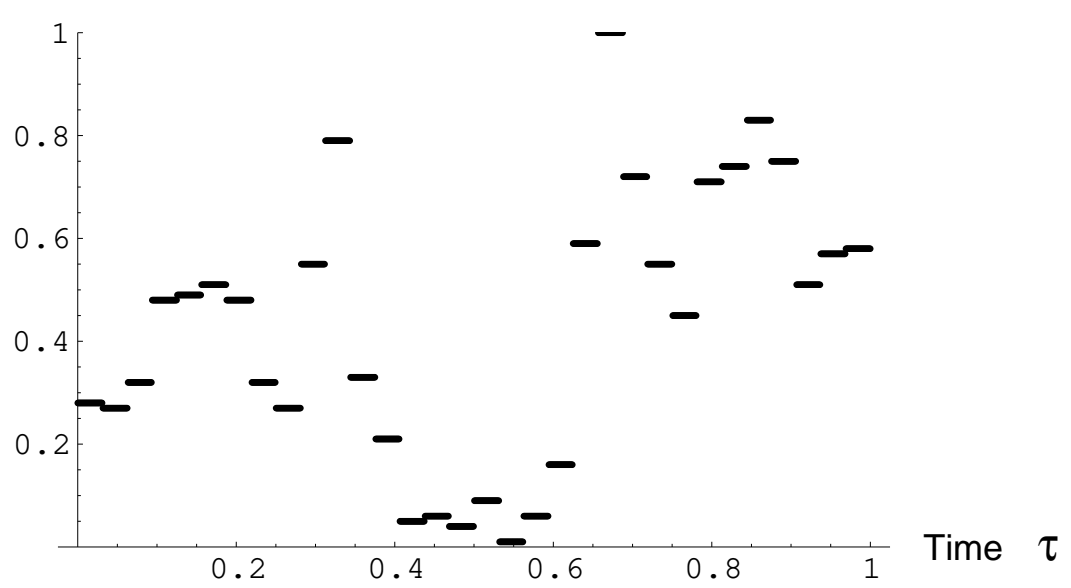


Figure 2c

Response of modified trigger system: running window

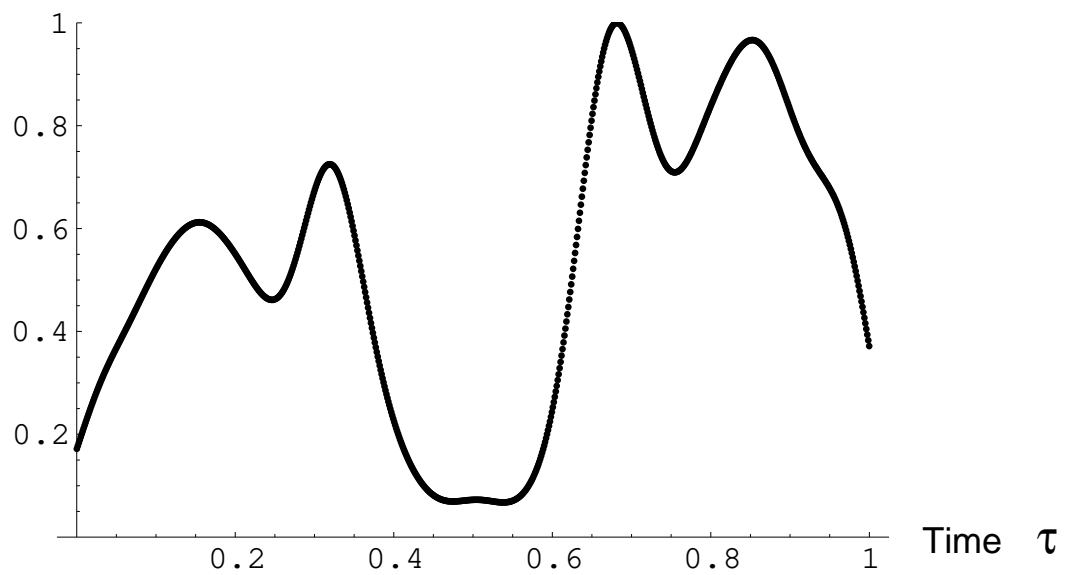


Figure 3

- $\quad$ Trigger system with bin averaging

- Trigger system with running window

- Unmodified trigger system

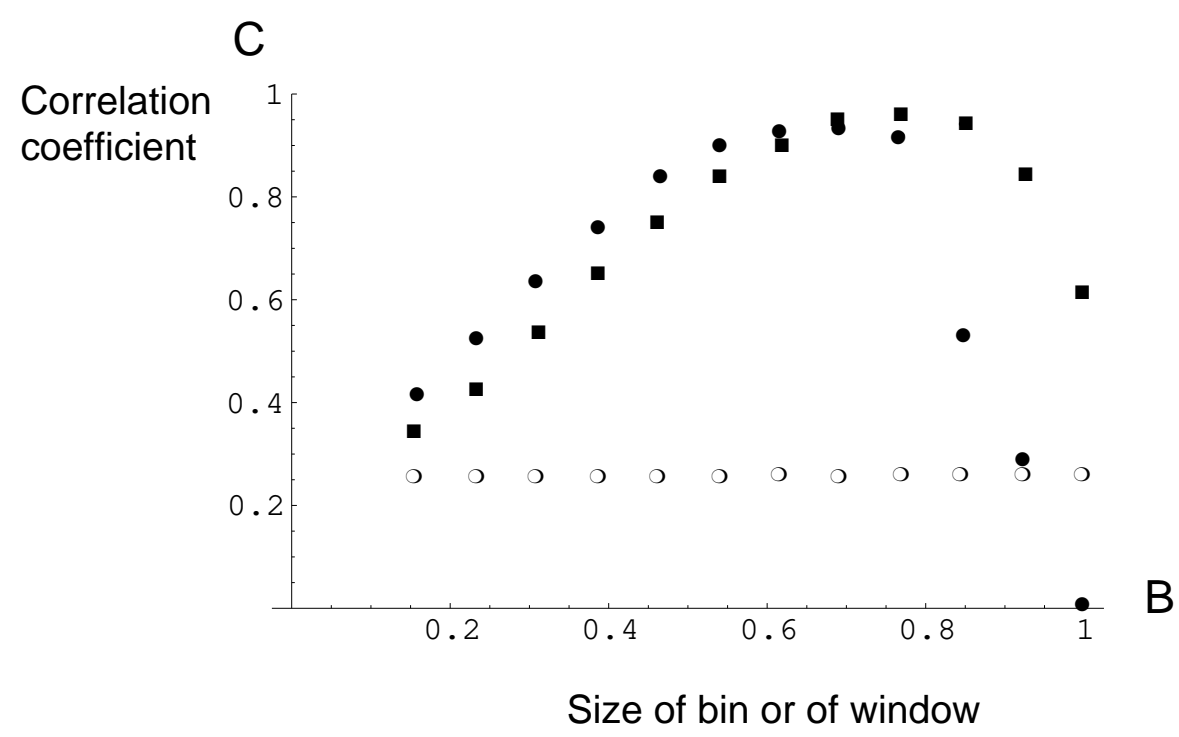


Figure 4

- Trigger system with bin averaging

- Trigger system with running window

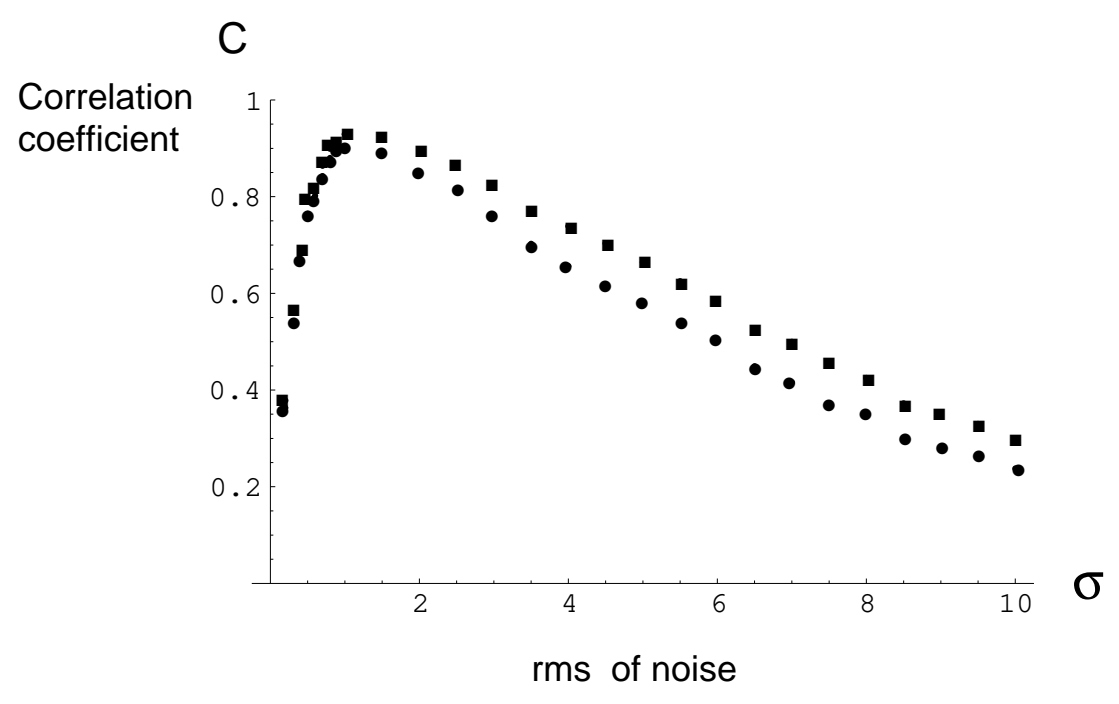

